\title{
Tratamiento del tabaquismo en los centros de drogodependencias y alcoholismo en España. Estado de la situación y perspectivas futuras.
}

\author{
Elisardo Becoña Iglesias ${ }^{(1)}$, Victoriana García García ${ }^{(2)}$, Jesús de Echave Sanz ${ }^{(3)}$, Juan José Fernández Miranda ${ }^{(4)}$, \\ M $^{\text {a Mar Sánchez Fernández }}{ }^{(5)}$, Antonio Terán Prieto ${ }^{(6)}$, Cristina Pinet Ogué(7), Jaume Serrano ${ }^{(8)}$, \\ Juan Carlos Rivera Aullol ${ }^{(9)}$, César Pereiro Gómez ${ }^{(10)}$, José Javier Moreno Arnedillo(11), \\ Francisco Javier Herrero García de Osma ${ }^{(12)}$ Javier Ruiz Fernández ${ }^{(13)}$ y Agurtzane Castillo ${ }^{(14)}$.
}

\author{
(1) Socidrogalcohol. Avda. Hospital Militar, 180. 08023 Barcelona. \\ (2) Victoriana García García. Unidad de Conductas Adictivas. Instituto Provincial de Bienestar Social. Córdoba. \\ (3) Jesús de Echave Sanz. Centro Municipal de Atención y Prevención de las Adicciones (CMAPA). Zaragoza. \\ (4)Juan José Fernández Miranda. Centro de Salud Mental SESPA. Avilés (Asturias). \\ (5) Mar Mar Sánchez Fernández. Equipo de Atención a Drogodependencias. Ciudad Real. \\ (6) Antonio Terán Prieto. Centro Ambulatorio de Tratamiento a Drogodependencias "San Juan de Dios". Palencia \\ (7) Cristina Pinet Ogué. Unidad de Conductas Adictivas. Hospital de la Santa Creu i Sant Pau. Barcelona. \\ (8) Jaume Serrano. CAS Horta-Ginardó (Barcelona). \\ (9) Juan Carlos Rivera Aullol. Unidad de Alcoholismo. Hospital Psiquiátrico de Plasencia (Cáceres). \\ (10)César Pereiro Gómez. Unidad Asistencial de Drogodependencias de A Coruña (ACLAD). A Coruña \\ (11) José Javier Moreno Arnedillo. Departamento de Prevención y Promoción de la Salud. Instituto de Salud Pública. Ayuntamiento de Madrid. \\ (12) Francisco Javier Herrero García de Osma. Departamento de Evaluación y Calidad. Instituto de Salud Pública. Ayuntamiento de Madrid. \\ (13) Javier Ruiz Fernández. Servicios Sociales. Ayuntamiento de Portugalete (Vizcaya). \\ (14) Agurtzane Castillo. Unidad de Conductas Adictivas. Castellón.
}

\section{RESUMEN}

Se analiza en una muestra de 142 centros, la mayoría de drogodependencias y algunos de alcoholismo, de 11 comunidades autónomas españolas, los programas que realizan para el tratamiento del tabaquismo. El 55.6\% llevan a cabo tratamiento de fumadores. A lo largo del año 2004 en dichos centros hubo una demanda de tratamiento por parte de 3.771 personas. Fueron tratadas unas 3.000 , finalizando el tratamiento la mitad de ellas. Los niveles de abstinencia indicados para el final del tratamiento como en los seguimientos a los 6 y 12 meses son buenos, en relación a los estudios clínicos publicados. El tratamiento más utilizado es el combinado (psicológico y farmacológico) seguido del psicológico. Suelen ser los distintos profesionales del equipo terapéutico los que llevan a cabo el tratamiento. Indican varias limitaciones para realizar este tratamiento, incluidos los presentes en cualquier adicción (ej., baja motivación de los fumadores), carencia de tiempo, poco personal, etc. Aprecian un claro incremento de la demanda, tanto de personas que acuden con solo dependencia de la nicotina como de sus usuarios clásicos que están a tratamiento por otras dependencias. Tienen clara su relevancia profesional en el tratamiento de esta adicción y la alta eficacia de los tratamientos que llevan a cabo con los fumadores. Ven necesario que se les aporten más medios para hacer frente al incremento continuo de demanda que están teniendo para el tratamiento de la dependencia de la nicotina en sus centros.

Palabras clave: Fumadores, dependencia, nicotina, centros, drogodependencias, alcoholismo, tratamiento, España.

\section{SUMMARY}

We analyzed in a sample of 142 Centers of Drug Abuse and Alcoholism, in 11 regions of Spain, the programs that they carry out for smoking cessation. The $55.6 \%$ of centers carries out treatment of smokers. Along the year 2004 in this centers had a treatment demand of 3,771 smokers, 3,000 were treated, concluding the treatment half of them. The levels of abstinence for the end of the treatment and in the 6- and 12-months followup are good, in relation to the published clinical studies. The most utilized treatment are the combined (psychological and pharmacological) followed by the psychological treatment. They are usually apply by diferent professionals as therapits. They indicate several limitations to carry out this treatment, included usual in any addiction (e.g., the smokers' low motivation), lack of time, limited personal, etc. They appreciates a clear increment of the demand, so much of people that they go with single nicotine dependence like of its classic users that are to treatment for other dependences. This data indicated the professional relevance of Centers of Drug Dependence in the treatment of nicotine dependence and the high effectiveness of the treatments that carry out with the smokers. They consider necessary that they have more means to make in front of the continuous increment of demand that they are having for the treatment of the nicotine dependence in their centers.

Keywords: smoking, dependence, nicotine, center, Drug Abuse, Alcoholism, treatment, Spain. 


\section{INTRODUCCIÓN}

E consumo de cigarrillos constituye en la actualidad un grave problema de salud pública. Estamos hablando de la primera causa evitable de morbi-mortalidad de los países desarrollados. De ahí que el interés por su control y su tratamiento haya sido, y sigue siendo, muy alto. La magnitud del problema, en cifras, es claro. En España mueren actualmente de modo prematuro por consumir cigarrillos en torno a 50.000-54.000 personas cada año (Banegas, Díez, González, Villar y Rodríguez-Artalejo, 2005; Montes, Pérez y Gestal, 2004). Aunque en los últimos años ha descendido la prevalencia del número de fumadores en España, todavía fuma el $31 \%$ de la población de 16 o más años (37.6\% varones y $24.7 \%$ mujeres) (Ministerio de Sanidad y Consumo, 2005). Y, el problema está en los niños y jóvenes que se van incorporando al consumo de tabaco, de modo cada vez mayor, especialmente en mujeres, por la agresiva publicidad que se dirige a ellos (Salvador, 1996).

La dependencia de la nicotina (American Psychiatric Association, 2000) está incluida en los sistemas diagnósticos bien conocidos que la clasifican dentro de la lista de sustancias psicoactivas capaces de generar dependencia. Por ello, su diagnóstico, características (ej., dependencia, síndrome de abstinencia, etc.), curso del tratamiento y recaída son semejantes a las demás sustancias adictivas (Jiménez et al., 2004). Quizás la diferencia más relevante es que, al igual que el alcohol, se trata de una droga legal que tiene detrás de sí una larga historia. En las últimas décadas, ante las claras consecuencias que acarrea en la salud, se han ido adoptando cada vez más medidas para su control. Y, estas medidas de control, implican también ofertar a los fumadores tratamientos para que puedan dejar de fumar (Becoña, 2004a).

Son distintos los abordajes y lugares donde se tratan a los fumadores. Nosotros aquí nos centramos únicamente en los Centros de Drogodependencias y Alcoholismo. Previamente, es preciso indicar que la creciente relevancia del tratamiento de los fumadores se asienta en dos realidades emergentes. Por una parte se ha incrementado significativamente la conciencia social sobre la gravedad del problema y con ello la puesta en marcha de medidas restrictivas al consumo, que a su vez han incrementado la demanda de tratamientos por parte de los fumadores. Por otra parte, se ha incrementado la disponibilidad de tratamientos eficaces para dejar de fumar, sean psicológicos (Becoña, 2004b), farmacológicos (Nerín y Córdoba, 2004; Sáiz, García-Portilla, Matínez, Bascarán, Paredes y Bobes, 2004) o combinados (Herrero y Moreno, 2004).

Los Centros de Drogodependencias disponen de un alto nivel de especialización en el tratamiento de los problemas de adicción relacionados con las drogas ilegales, especialmente de la heroína. Precisamente surgieron para paliar la epidemia de heroína surgida en España a partir de la década de los 70 y la creación del Plan Nacional sobre Drogas en 1985. En los últimos años las demandas de tratamiento de las distintas drogodependencias han variado ligeramente, con la estabilización de la demanda de heroína, el surgimiento creciente de demandas por dependencia de cocaína y en menor medida del cannabis (Plan Nacional sobre Drogas, 2003, 2004). También destaca en los últimos años el creciente peso que han tomado nuevos problemas como la comorbilidad (Mateu, Astals y Torréns, 2005) y la presencia del policonsumo, un problema que siempre ha existido en este tipo de pacientes con dependencia a otras drogas. La politoxicomanía siempre ha sido un problema clásico en en el ámbito clínico, pero que con la disponibilidad de nuevas drogas cada vez se ha hecho más relevante. Una de éstas drogas que siempre ha estado presente es la nicotina.

Las Unidades de Alcoholismo, con los distintos nombres que han ido recibiendo en las últimas décadas, llevan muchos años centradas en el tratamiento de las personas con dependencia del alcohol (SantoDomingo, 2002). Su creación, desarrollo, implantación y disponibilidad de recursos han sido siempre escasos si se considera la magnitud del problema en España. Al ser una droga socialmente aceptada no ha tenido el impacto que tuvo hace tres décadas la epidemia de la heroína en España, ni se han invertido tantos recursos como los dedicados a las drogas ilegales. En lo que se refiere al tema del tabaco, de modo semejante a lo que ha ocurrido en las Unidades Asistenciales de Drogodependencias, las Unidades de Alcoholismo han abierto en los últimos años el abanico de posibilidades terapéuticas a sus pacientes con problemas de comorbilidad (tanto psiquiátrica como referida a la dependencia de otras sustancias, e incluso a veces a adicciones comportamentales, como es el caso del juego patológico). Entre estas nuevas opciones terapéuticas se encuentra en muchos casos también la dependencia de la nicotina (Nieva, Gual, Ortega y Mondón, 2004).

Por tanto, hoy podemos hablar de un nuevo fenómeno, creciente desde hace unos años, como es el de las demandas de tratamiento para dejar de fumar, y la incipiente oferta específica de tratamiento de la dependencia nicotínica, llevada a cabo en los Centros de Drogodependencias y Unidades de Alcoholismo (Echave, Flordelís, García, de la Riba, Llopis y Martín, 2004).

La demanda, oferta, servicios, etc., para el tabaquismo depende en este momento de varios factores, desde si dicho tratamiento está o no en la cartera de servicios del centro o de los tratamientos ofrecidos en cada Comunidad Autónoma (ej., Andalucía, Valencia), a la capacitación e interés de los profesionales que ofertan asistencia en tabaquismo, o a la disponibilidad o no de centros donde se oferta este tipo de intervención. 
La realidad es que en este momento el tabaquismo, aparte de los problemas físicos bien conocidos, se relaciona con un amplio conjunto de trastornos, como la modificación del estado de ánimo, ansiedad, esquizofrenia, etc. (Gurrea y Pinet, 2004; Martínez, Gurpegui, Díaz y León, 2004), aparte del mayor consumo paralelo de otras sustancias psicoactivas. Así, del 60 al 100\% de los pacientes con dependencia de cannabis, cocaína o heroína fuman cigarrillos (Plan Nacional sobre Drogas, 2003).

Todo ello sugiere la necesidad de conocer cual es el estado de la situación del tratamiento para fumadores que se está desarrollando en estos centros, y cómo puede mejorarse. Al tiempo, es preciso valorar qué posibilidades hay de que los Centros de Drogodependencias y Alcoholismo se impliquen más en llevar a cabo tratamiento del tabaquismo para todos sus pacientes con dependencia de drogas ilegales; y, explorar necesidades para la puesta en marcha de tratamientos específicos para otros pacientes o para las personas de la población general con dependencia de la nicotina.

\section{OBJETIVOS}

Los objetivos del presente estudio son: 1) conocer la situación en los Centros de Drogodependencias y Unidades de Alcoholismo en relación al tratamiento de la dependencia de la nicotina; 2) conocer el tipo de tratamiento realizado y resultados obtenidos en aquellos Centros y Unidades donde se lleva a cabo dicho tratamiento; y 3) conocer las perspectivas de futuro que consideran que va a tener este tipo de tratamiento, tanto en sus pacientes dependientes a drogas ilegales como en otros posibles tipos de pacientes. Aunque lo ideal sería obtener información de todos los centros de drogodependencias y alcoholismo existentes en nuestro país, los medios disponibles para el presente estudio no permiten esta opción. Por tanto, este estudio constituye una primera aproximación a la realidad del tratamiento del tabaquismo en el sector que atiende a otras drogodependencias.

\section{MÉTODO}

A través de un total de 11 coordinadores, uno por cada comunidad autónoma que participaron en el estudio (Andalucía, Aragón, Asturias, Castilla-La Mancha, Castilla-León, Cataluña, Extremadura, Galicia, Madrid, País Vasco y Valencia), se envió un cuestionario a los distintos centros de drogodependencias y/o alcoholismo (en algunas comunidades se tratan conjuntamente personas con dependencia del alcohol o de otras drogas, o bien las unidades están vinculadas funcionalmente u orgánicamente a las drogodependencias). Se obtuvo un total de 142 cuestionarios válidos de otros tantos centros de drogodependencias y algunos de alcoholismo, del total de 276 centros a los que se les envió el cuestionario (tabla 1), con una respuesta promedio del 51,45\%, aunque en algunas comunidades llegó a contestar el 100\%.

El cuestionario utilizado en el presente estudio incluye preguntas abiertas y cerradas en las que se les preguntaba a los responsables de cada centro distintas cuestiones referidas a si en su centro realizan o no tratamiento para los fumadores y, en caso de hacerlo, qué tipo de tratamiento utilizan, resultados obtenidos, así como varias preguntas abiertas para conocer cómo ven el futuro del tratamiento de los fumadores.

Los datos cuantitativos solicitados se referían siempre a las personas atendidas o tratadas desde el 1 de enero hasta el 31 de diciembre del año 2004.

Tabla 1. Distribución de la muestra de Centros de Drogodependencias y/o Alcoholismo en cada una de las Comunidades Autónomas españolas que participaron en el estudio.

\begin{tabular}{|lccc|}
\hline Comunidad Autónoma & $\begin{array}{c}\text { Cuestionarios } \\
\text { enviados }\end{array}$ & $\begin{array}{c}\text { Cuestionario } \\
\text { recibidos }\end{array}$ & $\begin{array}{c}\text { Cuetionarios de } \\
\text { solo centros de } \\
\text { drogodependencias }\end{array}$ \\
\hline Andalucía & 79 & 6 & No \\
Aragón & 14 & 12 & Sí \\
Asturias & 11 & 11 & Sí \\
Castilla-La Mancha & 7 & 6 & Sí \\
Castilla-León & 5 & 5 & Sí \\
Cataluña & 59 & $51(1)$ & Sí \\
Extremadura & 8 & 2 & No \\
Galicia & 13 & 13 & Sí \\
Madrid & 30 & 10 & Sí \\
País Vasco & 22 & 16 & Sí \\
Valencia & 28 & 10 & Sí \\
\hline Total & 276 & 142 & \\
\hline
\end{tabular}

(1) Aquí contestó el $100 \%$ dado que los 51 cuestionarios recibidos corresponden a los equipos de los 59 centros que existen administrativamente, dado que hay centros que constan como distintos pero tienen el mismo equipo. 


\section{RESULTADOS}

\section{Demanda de tratamiento y fumadores evaluados $y$ tratados}

El 55.6\% de los centros o unidades indican que llevan a cabo algún tipo de tratamiento de fumadores. De éstos, la gran mayoría aporta datos sobre las características de las personas que han tratado, su número y tipo de tratamiento aplicado. Así, el número de personas que demandaron tratamiento para dejar de fumar, o a las que se les recomendó hacerlo, varía mucho en número de unos centros a otros. En la tabla 2 indicamos la agrupación de las demandas por tamaño de la demanda. Allí se puede ver que la mayoría de los centros, el 45.7\%, tratan de 1 a 15 fumadores por año. Pero también destaca en el lado opuesto que hay un centro que tiene una demanda de tratamiento de 663 fumadores al año, mientras casi un $20 \%$ de los centros tiene una demanda de tratamiento que oscila entre 100 y 300 fumadores al año.

Dado que no es lo mismo demandar tratamiento, o remitir a una persona al mismo, que ofertar dicho tratamiento, hemos analizado también qué personas de las que demandaron asistencia para dejar de fumar fueron evaluadas y cuáles realizaron efectivamente el tratamiento. Dos de los centros que han sugerido a algún fumador ser tratado no contestan a esta cuestión porque debido a su bajo número no llegaron a hacer la evaluación.

En relación con el número de personas que han sido evaluadas (tabla 2), los resultados son semejantes a los anteriores. Así, el $51.4 \%$ de los centros ha evaluado de 1 a 15 fumadores, el 11,8\% entre 16 y
30, destacando que el $16.2 \%$ ha evaluado de 51 a 100 fumadores. También destaca que sólo 9 centros han evaluado a más de 100 personas cada uno, mientras un solo centro ha evaluado a 515 fumadores a lo largo de un año. Para las personas tratadas, los resultados son semejantes a los indicados para las personas evaluadas (tabla 2) ya que aquí han tenido poca pérdida de sujetos, al menos para quienes han realizado como mínimo una sesión de tratamiento.

Para entender mejor los datos anteriores, los centros que han contestado, tomados en su conjunto, indican que un total de 3.771 personas han demandado tratamiento en su Centro para dejar de fumar o ellos les han sugerido dejar de fumar. Entre ellas, 3.109 han pasado por la evaluación previa al tratamiento, y 2.948 han realizado una o más sesiones del tratamiento a lo largo del año 2004.

Dejar de fumar, como cualquier otra conducta adictiva, presenta una característica bien conocida en los tratamientos de las adicciones: la pérdida de sujetos a lo largo del mismo. Por este motivo hemos analizado también las personas que han finalizado el tratamiento, considerando los que lo han comenzado. De este modo vemos que mientras que 67 centros contestaron con datos sobre la evaluación, baja a 55 los que contestaron con datos sobre los fumadores que finalizaron el tratamiento (tabla 3). Aquí se aprecia que la mayoría de los centros, el $60 \%$, indica que finalizan el tratamiento entre 1 y 15 fumadores, aunque también destaca que en tres centros lo finalizan entre 100 y 150 fumadores. De los datos que nos proporcionan los centros, el número global que obtenemos de los fumadores que han finalizado el tratamiento fue de 1.355 fumadores.

Tabla 2. Personas que demandaron tratamiento parar dejar de fumar o se les sugirió hacerlo, las que fueron evaluadas y las que fueron tratadas

\begin{tabular}{|lcccccc|}
\hline & $\begin{array}{c}\text { Personas que demandan } \\
\text { o se le propone } \\
\text { tratamiento }\end{array}$ & \multicolumn{2}{c}{ Personas } & \multicolumn{2}{c|}{$\begin{array}{c}\text { Personas } \\
\text { tratadas }\end{array}$} \\
Número de personas & $\mathbf{n}$ & $\%$ & $\mathbf{n}$ & $\%$ & $\mathbf{n}$ & $\%$ \\
\hline De 1 a 15 & 32 & 45.7 & 35 & 51,5 & 34 & 50,7 \\
De 16 a 30 & 13 & 18,6 & 8 & 11,8 & 9 & 13,4 \\
De 31 a 50 & 2 & 2,8 & 5 & 7,3 & 8 & 11,9 \\
De 51 a 100 & 9 & 12,8 & 11 & 16,2 & 7 & 10,4 \\
De 100 a 150 & 8 & 11,4 & 5 & 7,3 & 5 & 7,5 \\
De 151 a 200 & 3 & 4,3 & 2 & 2,9 & 2 & 3,0 \\
De 201 a 300 & 2 & 2,8 & 1 & 1,5 & 1 & 1,5 \\
Más de 300 & 1 & 1,4 & 1 & 1,5 & 1 & 1,5 \\
\hline Total Centros & 70 & 100 & 68 & 100 & 67 & 100 \\
\hline
\end{tabular}


Tabla 3. Personas que han acabado el tratamiento para dejar de fumar, partiendo de los que han

comenzado el mismo

\begin{tabular}{|lcc|}
$\begin{array}{l}\text { Personas que finalizan } \\
\text { el tratamiento }\end{array}$ & $\mathbf{n}$ & $\%$ \\
\hline De 1 a 15 & 33 & 60,0 \\
De 16 a 30 & 10 & 18,2 \\
De 31 a 50 & 2 & 3,6 \\
De 51 a 100 & 7 & 12,7 \\
De 101 a 150 & 3 & 5,5 \\
\hline Total & 55 & 100 \\
\hline
\end{tabular}

Tabla 4. Demanda de tratamiento y realización del mismo a lo largo del año 2004

\begin{tabular}{lcc|} 
& $\mathbf{N}^{\circ}$ centros & $\mathbf{n}$ \\
\hline Demanda de tratamiento & 70 & 3.771 \\
Realizan la evaluación & 68 & 3.109 \\
Personas tratadas & 67 & 2,948 \\
Personas que finalizan el tratamiento & 55 & 1.355 \\
\hline
\end{tabular}

\section{Porcentaje de abstinencia al final del tratamiento}

Otro dato de interés es conocer el nivel de abstinencia que logran los fumadores en tratamiento, donde la mayoría de ellos son usuarios de los centros de drogodependencias, aunque algunos centros también tratan a personas de la población general o a pacientes remitidos desde atención primaria.

Lo primero a indicar es que una parte importante de los centros no lleva un registro sistemático de la abstinencia de sus fumadores, lo que impide tener datos de todos los centros. Así, de los centros que contestan sobre los fumadores que logran la abstinencia al final del tratamiento, considerando a los que han acudido al menos a una sesión de tratamiento, son 34 (el $24 \%$ del total) los que contestan (tabla 5), que baja a 21 centros (el 14.8\%) los que indican datos de eficacia (tabla 6). La mayoría de los centros, el 64.7\%, indica que de 1 a 15 fumadores finalizan el tratamiento, un $14.7 \%$ de 16 a 30 , un $11.7 \%$ de 31 a 50 y el $8.8 \%$ entre 51 y 100 . A nivel de porcentaje de abstinencia la mayoría, el $38.1 \%$, indica que el nivel que obtienen está entre el 41 y el $50 \%$ al final del tratamiento, aunque hay porcentajes de abstinencia en la parte baja del
11 al 20\%, como en la alta, del 91 al 100\%. Aunque este es el dato real, si hacemos el promedio de todos los centros, nos encontramos que el porcentaje de abstinencia que obtienen al final, de estos centros que nos proporcionan sus datos, está entre el 40 y el $50 \%$ al final del tratamiento, considerando a todos los fumadores que han acudido a una o a más sesiones de tratamiento.

Otro modo de evaluar la eficacia del tratamiento es conocer el número de fumadores abstinentes entre quienes han finalizado el mismo. Este es un criterio distinto al anterior y más laxo, pero que refleja en muchas ocasiones mejor la realidad asistencial. Con este criterio, en la tabla 5 indicamos el número de personas que logran la abstinencia y en la tabla 6 el porcentaje de abstinencia. En este último caso, el número de centros que contesta es mayor, 35 y 27 respectivamente, y los porcentajes de abstinencia que indican son buenos. Así, mientras que en un $38,1 \%$ de los centros indica que dejan de fumar entre un 41 y un $50 \%$ de los casos, un $29.2 \%$ de los centros señala que consiguen la abstinencia entre el 51 y el 100\%. de los pacientes tratados. 
Tabla 5. Personas que logran la abstinencia al final del tratamiento, de aquellos que han acudido al menos a una sesión de tratamiento y de aquellos que han finalizado el tratamiento

\begin{tabular}{|c|c|c|c|c|}
\hline \multirow[b]{2}{*}{ Abstinentes } & \multicolumn{2}{|c|}{$\begin{array}{l}\text { Abstinentes que acuden } \\
\text { al menos a } 1 \text { sesión }\end{array}$} & \multicolumn{2}{|c|}{$\begin{array}{c}\text { Abstinentes que } \\
\text { finalizan tratamiento }\end{array}$} \\
\hline & $n$ & $\%$ & $n$ & $\%$ \\
\hline De 1 a 15 & 22 & 64,7 & 23 & 65,7 \\
\hline De 16 a 30 & 5 & 14,7 & 5 & 14,3 \\
\hline De 31 a 50 & 4 & 11,7 & 2 & 5,7 \\
\hline De 51 a 100 & 3 & 8,8 & 3 & 8,6 \\
\hline De 101 a 150 & 0 & 0,0 & 2 & 5,7 \\
\hline Total & 34 & 100 & 35 & 100 \\
\hline $\begin{array}{l}\text { Total de personas que } \\
\text { logran la abstinencia: }\end{array}$ & \multicolumn{2}{|c|}{634} & \multicolumn{2}{|c|}{807} \\
\hline
\end{tabular}

\begin{tabular}{|c|c|c|c|c|}
\hline \multirow[b]{2}{*}{ \% de abstinencia } & \multicolumn{2}{|c|}{ Al menos 1 sesión } & \multicolumn{2}{|c|}{ Han finalizado tratamiento } \\
\hline & $\mathbf{n}$ & $\%$ & $n$ & $\%$ \\
\hline Del $11 \%$ al $20 \%$ & 3 & 14,3 & 1 & 3,7 \\
\hline Del $21 \%$ al $30 \%$ & 3 & 14,3 & 4 & 14,8 \\
\hline Del $31 \%$ al $40 \%$ & 1 & 4,8 & 2 & 7,4 \\
\hline Del $41 \%$ al $50 \%$ & 8 & 38,1 & 7 & 25,9 \\
\hline Del $51 \%$ al $60 \%$ & 3 & 14,3 & 3 & 11,1 \\
\hline Del $61 \%$ al $70 \%$ & 1 & 4,8 & 0 & 0,0 \\
\hline Del $71 \%$ al $80 \%$ & 1 & 4,8 & 3 & 11,1 \\
\hline Del $81 \%$ al $90 \%$ & 0 & 0,0 & 4 & 14,8 \\
\hline Del $91 \%$ al $100 \%$ & 1 & 4,8 & 3 & 11,1 \\
\hline Total & 21 & 100 & 27 & 100 \\
\hline
\end{tabular}

Tabla 7. Personas que están abstinentes a los 6 meses, de aquellos que han acudido al menos a una sesión de tratamiento y de los que han finalizado el tratamiento

\begin{tabular}{|c|c|c|c|c|}
\hline \multirow{2}{*}{$\begin{array}{l}\text { Personas abstinentes } \\
\text { a los } 6 \text { meses }\end{array}$} & \multicolumn{2}{|c|}{ Al menos 1 sesión } & \multicolumn{2}{|c|}{ Finalizaron tratamiento } \\
\hline & & $\%$ & n & $\%$ \\
\hline De 1 a 15 & 16 & 76.2 & 22 & 84.6 \\
\hline De 16 a 30 & 3 & 14.3 & 3 & 11.5 \\
\hline De 31 a 50 & 1 & 4.8 & 1 & 3,8 \\
\hline \multirow[t]{2}{*}{ De 51 a 100} & 1 & 4.8 & 0 & 0.0 \\
\hline & 21 & 100 & 26 & 100 \\
\hline $\begin{array}{l}\text { Total de personas que } \\
\text { logran la abstinencia ( } 6 \text { meses) }\end{array}$ & & & & \\
\hline
\end{tabular}


Tabla 8. Porcentajes de personas que están abstinentes a los 6 meses, de aquellos que han acudido al menos a una sesión de tratamiento y de los que han finalizado el tratamiento

\begin{tabular}{|c|c|c|c|c|}
\hline \multirow[b]{2}{*}{$\%$ de abstinencia } & \multicolumn{2}{|c|}{ Al menos 1 sesión } & \multicolumn{2}{|c|}{ Finalizaron tratamiento } \\
\hline & & $\%$ & $\mathbf{n}$ & $\%$ \\
\hline Del $11 \%$ al $20 \%$ & 1 & 7.7 & 1 & 5.0 \\
\hline Del $21 \%$ al $30 \%$ & 2 & 15.4 & 2 & 10.0 \\
\hline Del $31 \%$ al $40 \%$ & 4 & 30.8 & 4 & 20.0 \\
\hline Del $41 \%$ al $50 \%$ & 3 & 23.1 & 5 & 25.0 \\
\hline Del $51 \%$ al $60 \%$ & 1 & 7.7 & 2 & 10.0 \\
\hline Del $61 \%$ al $70 \%$ & 0 & 0.0 & 2 & 10.0 \\
\hline Del $71 \%$ al $80 \%$ & 2 & 15.4 & 1 & 5.0 \\
\hline Del $81 \%$ al 90\% & 0 & 0.0 & 1 & 5.0 \\
\hline Del $91 \%$ al $100 \%$ & 0 & 0.0 & 2 & 10.0 \\
\hline Total & 13 & 100 & 20 & 10 \\
\hline
\end{tabular}

Tabla 9. Personas que están abstinentes a los 12 meses, de aquellos que han acudido al menos a una sesión de tratamiento y de los que han finalizado el tratamiento

\begin{tabular}{|c|c|c|c|c|}
\hline \multirow{2}{*}{$\begin{array}{l}\text { Personas abstinentes } \\
\text { a los } 12 \text { meses }\end{array}$} & \multicolumn{2}{|c|}{ Al menos 1 sesión } & \multicolumn{2}{|c|}{ Finalizaron tratamiento } \\
\hline & & $\%$ & $\mathbf{n}$ & $\%$ \\
\hline Ninguno & 1 & 6.7 & 17 & 94.0 \\
\hline De 1 a 15 & 13 & 86.7 & 0 & 0.0 \\
\hline De 16 a 30 & 0 & 0.0 & 0 & 0.0 \\
\hline De 31 a 50 & 1 & 6.7 & 1 & 5.6 \\
\hline Total & 21 & 100 & 26 & 100 \\
\hline $\begin{array}{l}\text { Total de personas que } \\
\text { logran la abstinencia ( } 12 \text { meses) }\end{array}$ & \multicolumn{2}{|c|}{168} & & \\
\hline
\end{tabular}

Tabla 10. Porcentajes de personas que están abstinentes a los 12 meses, de aquellos que han acudido al menos a una sesión de tratamiento y de los que finalizaron el tratamiento

\begin{tabular}{|c|c|c|c|c|}
\hline \multirow[b]{2}{*}{$\%$ de abstinencia } & \multicolumn{2}{|c|}{ Al menos 1 sesión } & \multicolumn{2}{|c|}{ Finalizaron tratamiento } \\
\hline & $\mathbf{n}$ & $\%$ & $\mathbf{n}$ & $\%$ \\
\hline Del $11 \%$ al $20 \%$ & 0 & 0.0 & 2 & 12.5 \\
\hline Del $21 \%$ al $30 \%$ & 3 & 30.0 & 2 & 12.5 \\
\hline Del $31 \%$ al $40 \%$ & 2 & 20.0 & 5 & 31.3 \\
\hline Del $41 \%$ al $50 \%$ & 2 & 20.0 & 2 & 12.5 \\
\hline Del $51 \%$ al $60 \%$ & 2 & 20.0 & 1 & 6.2 \\
\hline Del $61 \%$ al $70 \%$ & 0 & 0.0 & 1 & 6.2 \\
\hline Del $71 \%$ al $80 \%$ & 1 & 10.0 & 1 & 6.2 \\
\hline Del $81 \%$ al $90 \%$ & 0 & 0.0 & 1 & 6.2 \\
\hline Total & 10 & 100 & 16 & 100 \\
\hline
\end{tabular}


Tabla 11. Ejemplo de los resultados de cuatro centros. Demanda de tratamiento y evolución del tratamiento.

\begin{tabular}{|c|c|c|c|c|c|c|c|c|c|c|}
\hline \multirow[b]{2}{*}{ Centro } & \multirow{2}{*}{$\begin{array}{l}\mathrm{N}^{\circ} \text { personas } \\
\text { demandan } \\
\text { tratamiento }\end{array}$} & \multirow[b]{2}{*}{$\begin{array}{c}\mathbf{N}^{\circ} \text { personas } \\
\text { evaluadas }\end{array}$} & \multirow[b]{2}{*}{$\begin{array}{c}\mathbf{N}^{\circ} \text { personas } \\
\text { tratadas }\end{array}$} & \multirow{2}{*}{$\begin{array}{c}\text { Finalizan } \\
\text { tratamiento }\end{array}$} & \multicolumn{6}{|c|}{$\mathbf{N}^{\circ}$ de personas abstinentes al } \\
\hline & & & & & & $\begin{array}{l}\text { ial } \\
(2)\end{array}$ & $\begin{array}{r}6 \mathrm{~m} \\
(1)\end{array}$ & $\begin{array}{l}\text { eses } \\
\text { (2) }\end{array}$ & $\begin{array}{r}12 \mathrm{~m} \\
\text { (1) }\end{array}$ & $\begin{array}{l}\text { ieses } \\
\text { (2) }\end{array}$ \\
\hline 1 & 15 & 10 & 8 & 7 & - & 7 & - & 7 & - & 7 \\
\hline 2 & 58 & 47 & 41 & 20 & 21 & 18 & - & - & - & - \\
\hline 3 & 159 & 140 & 135 & 75 & 72 & 72 & 90 & - & - & - \\
\hline 4 & 663 & 515 & 515 & - & - & - & - & - & - & - \\
\hline
\end{tabular}

Tabla 12. Ejemplo de los resultados de cuatro centros. Datos proporcionados de abstinencia.

\begin{tabular}{|c|c|c|c|c|c|c|c|c|}
\hline \multirow[b]{3}{*}{ Centro } & \multirow{3}{*}{$\begin{array}{c}\mathbf{N}^{\circ} \text { personas } \\
\text { tratadas }\end{array}$} & \multirow{3}{*}{$\begin{array}{c}\text { Finalizan } \\
\text { tratamiento }\end{array}$} & \multirow{2}{*}{\multicolumn{2}{|c|}{$\begin{array}{c}\text { Final del } \\
\text { tratamiento }\end{array}$}} & \multicolumn{4}{|c|}{$\%$ de abstinencia a los } \\
\hline & & & & & \multicolumn{2}{|c|}{6 meses } & \multicolumn{2}{|c|}{12 meses } \\
\hline & & & (1) & (2) & (1) & (2) & (1) & (2) \\
\hline 1 & 8 & 7 & - & 87 & - & 87 & - & 87 \\
\hline 2 & 41 & 20 & - & - & - & - & - & - \\
\hline 3 & 135 & 75 & 50 & 50 & - & - & - & 50 \\
\hline 4 & 515 & - & - & - & 35 & - & - & - \\
\hline
\end{tabular}

(1) Acudieron a 1 o más sesiones de tratamiento.

(2) Datos de los que finalizaron el tratamiento

\section{Porcentaje de abstinencia a los 6 y 12 meses de seguimiento}

Disponemos de datos de 21 centros (el 14.8\%) para conocer el número de fumadores que mantienen la abstinencia a los 6 meses y de 13 centros (el $9.2 \%$ ) que indican porcentajes de abstinencia a los 6 meses, referidos tanto a pacientes que han acudido a una sesión de tratamiento y como ha pacientes que han finalizado el tratamiento (tablas 7 y 8). De igual modo, disponemos de los datos de seguimiento de 12 meses en relación con los pacientes que han acudido a una sesión de tratamiento y de los que han acabado el tratamiento (tablas 9 y 10), aunque en este caso contestan pocos centros, entre 10 y 20.

A nivel de abstinencia, a los 6 y 12 meses, la mayoría están en el rango de abstinencia del 21 al 50\%. Sin embargo, el bajo número de centros que han contestado hace poco consistente este resultado. De confirmarse en más centros sería un resultado alto a nivel de abstinencia en este período temporal de seguimiento.

Como ejemplo de los datos proporcionados por los distintos centros, en las tablas 11 y 12 indicamos los datos de cuatro centros, con incremento de unos a otros en el número de fumadores tratados, para darnos una idea de los datos proporcionados por la mayoría de los centros y los resultados que obtienen. Como se ejemplifica en las citadas tablas, la mayoría de cen- tros no aporta datos de seguimiento. Esto viene dado por el hecho de que no tienen habitualmente capacidad para analizar los datos ya que su objetivo y labor es fundamentalmente terapéutica y la mayoría de sus esfuerzos se dedican a la asistencia.

\section{Satisfacción con el servicio recibido}

Los centros que contestaron a la valoración del servicio recibido por parte de los usuarios de los tratamiento para dejar de fumar (un total de 51) consideran la atención recibida como buena $(66.7 \%)$ o muy buena $(33.3 \%)$. En ningún caso se valora como regular o mala. A pesar de que es una evaluación sobre el propio trabajo, dicha respuesta indica claramente que los clínicos evalúan positivamente la percepción del trabajo que realizan y como lo ven en sus usuarios.

\section{Tipo de usuarios a los que se aplica el tratamiento}

La pregunta referida a los tipos de usuarios a los que les aplican el tratamiento tenía tres alternativas de respuesta:

a) Pacientes que están a tratamiento en el Centro por otras drogodependencias.

b) Pacientes que acuden al Centro sólo para dejar de fumar cigarrillos. 
c) Se llevan a cabo los tratamientos fuera del Centro (especificar donde y tipo de personas atendidas).

d) Otros (especificar).

Las respuesta de los centros que contestan se agrupa básicamente en las alternativas a) y b), ocurriendo que muchos de los centros tienen tanto pacientes de a) como b). Así, la mayoría de ellos tratan a los pacientes de sus Centros $(n=67)$ que acuden por problemas de dependencia de heroína, cocaína, cannabis, etc. y, con frecuencia, también a los pacientes que acuden a su centro específicamente para dejar de fumar $(n=54)$. En un grado menor, pero no despreciable, algunos centros ( $n=11$ ) también llevan a cabo tratamientos en centros de salud, centros de atención primaria, hospitales, centros municipales, en profesionales sanitarios y en empresas, bien por demanda, por dependencia funcional o por oferta de servicios externos.

\section{Tabla 13. Tipos de pacientes o usuarios a los que se atienden para tratar la dependencia de la nicotina}

\section{Tipo de pacientes o usuarios}

a) Pacientes que están a tratamiento en el Centro por otras drogodependencias $\mathbf{N}^{\circ}$ de centros

b) Pacientes que acuden al Centro sólo para dejar de fumar cigarrillos

c) Se llevan a cabo los tratamientos fuera del Centro
67

54

11

Nota: Los centros pueden contestar a 1, 2 ó 3 de las anteriores alternativas.

\section{Tipo de tratamiento que se aplica y quien lo aplica}

Dentro de los tratamientos eficaces hay dos tipos claros para utilizar en la clínica, los psicológicos y los farmacológicos junto a la combinación de ambos. Por ello les pedíamos que nos indicasen qué tipo de tratamiento utilizaban, si lo tenían protocolizado, y que nos proporcionasen información sobre el mismo. El tratamiento más utilizado por los distintos centros de drogodependencias y alcoholismo es el combinado ( $n=67)$, luego le sigue sólo psicológico $(n=9)$, segui- do en menor grado de otros (ver tabla 14). En varios casos del tratamiento combinado especifican que el que aplican va a depender de las características de cada paciente y que, en función de ello, se aplica tratamiento psicológico, médico o combinado. Por ello, en la mayoría de los casos, adecuan el tratamiento al paciente concreto y a sus características. En muchos casos los tratamientos están protocolizados. En otros, aunque indican que son tratamientos combinados, incluyen varios abordajes, tanto médicos, psicológicos, como otros.

Tabla 14. Tipo de tratamiento que se aplica en los Centros para tratar a los fumadores.

\begin{tabular}{|lcc|}
\hline Tipo de tratamiento aplicado & $\mathbf{n}$ & $\%$ \\
\hline Combinado & 67 & 82.7 \\
Psicológico & 9 & 11.1 \\
Consejo & 2 & 2.5 \\
Médico & 1 & 1.2 \\
Psicoeducativo & 1 & 1.2 \\
No especificado o sin protocolo & 1 & 1.2 \\
\hline Total & 81 & 100 \\
\hline
\end{tabular}

Respecto a quién lleva a cabo el tratamiento, si todo el equipo o algún profesional en concreto, la respuesta mayoritaria de los centros es que la realizan varios profesionales, habitualmente el psicólogo y el médico, junto al resto de los profesionales que forman los centros de drogodependencias $(n=54)$. En la mayoría de los casos, cuando hablan de varios profesionales, suele ser el psicólogo el que lleva el peso de las sesiones de tratamiento, ocurriendo en muchos casos que el médico recomienda el fármaco para dejar de fumar (TSN o bupropión) y sigue el resto de la terapia el psicólogo. Le sigue a continuación como profesional único que aplica el tratamiento el psicólogo $(n=17), y$ en menor grado sólo el médico $(n=4)$, o el médico y personal de enfermería, auxiliar de clínica o educador $(n=5)$. En un caso es el trabajador social el que aplica el tratamiento. 


\begin{tabular}{|lcc|}
\hline & Tabla 15. Quien Ileva a cabo el tratamiento & \\
\hline Aplica el tratamiento & $\mathbf{n}$ & \% \\
\hline Psicólogo & 17 & 21.0 \\
Médico & 4 & 4.9 \\
Médico y personal de enfermería & 3 & 3.7 \\
Médico y auxiliar de clínica & 1 & 1.2 \\
Médico y educador & 1 & 1.2 \\
Trabajador social & 1 & 1.2 \\
\hline Total & 81 & 100 \\
\hline
\end{tabular}

\section{Tiempo dedicado al tratamiento}

Las contestaciones referidas al tiempo dedicado al tratamiento de los fumadores se pueden agrupar en dos grandes grupos. El primero es el de aquellos centros que consideran que tienen carencia de personal para un adecuado tratamiento de la demanda recibida de fumadores, sean de sus usuarios o de potenciales nuevos usuarios. En algunos de estos casos, se asigna un tiempo específico para el tratamiento, incluso a veces por las tardes. El segundo es el de aquellos centros donde el tratamiento de los fumadores es ya otra actividad más de los mismos, al considerar a la dependencia de la nicotina como otra adicción más.

Para ejemplificar lo anterior, a continuación indicamos algunos ejemplos extraídos de cuatro centros:

- Centro 25. Habitualmente se dedican 1 psicólogo, 1 médico o un ATS, pero es variable dependiendo de las posibilidades reales de cada momento, lo que supone recurrir a otros profesionales puntualmente, o realizar el tratamiento sólo un terapeuta. El horario de los grupos es de tarde: 17 y 19 h.; y de mañanas a las $11 \mathrm{~h}$., con una sesión semanal. Se realiza cuando hay suficiente demanda y se da la posibilidad de darle respuesta. El tratamiento puede ser individual, grupal o mixto. El grupal consta de 7 sesiones semanales de 90 minutos. Hay un grupo abierto de continuidad y apoyo una vez al mes para los pacientes que ya han concluido las sesiones. El tratamiento individual es más elástico, tanto para iniciarlo como por el contenido de las sesiones, y a veces por tratarse de pacientes con problemática añadida.

- Centro 84. Las fases del tratamiento son: 1) acogida-demanda: trabajadora social, 2) deshabituación (tratamiento psicológico): psicóloga. El tratamiento se realiza tanto de modo individual como grupal. Depende de la disponibilidad horaria de los usuarios y sobre todo de la psicopatología asociada. Y, 3) tratamiento médico: cuando es necesario lo hace cualquiera de los médicos de la unidad. Se da consejo médico (información sobre el tabaco, plantearse disminuir el consumo) en casi todos los casos.
Las sesiones individuales son de una hora aproximadamente cada 15 días por la mañana (9-15 h.). Las sesiones grupales de 2 horas cada 15 días. Durante unos 6 meses aproximadamente, por la tarde (17-19 h.). Una vez finalizado el grupo se hace un seguimiento del mantenimiento de la abstinencia bien personal o telefónico al mes, 3, 6 y 12 meses.

- Centro 91. Se realiza igual que el de otras adicciones. Tras la valoración por los diferentes miembros del equipo: médico, psicólogo y trabajador social, solicitadas las pruebas analíticas complementarias y firmado el contrato terapéutico se inicia el tratamiento. Recae fundamentalmente en el médico y psicólogo con intervenciones puntuales de enfermería. No hay un horario o un día establecido para el tratamiento de estos pacientes, sino el que corresponde por la cita. La dedicación y tiempo es poco importante al tratarse de pocos pacientes.

- Centro 137. Las fases son las siguientes. 1) realización de una primera visita, a poder ser médico y psicólogo, 2) pauta de farmacología según se acuerde, 3) entrevista con enfermería, 4) en adelante, visitas de seguimiento por parte de médico o psicólogo para ir valorando el proceso de abstinencia o recaídas. No hay un día o un horario fijo

\section{Problemas que se encuentran en el tratamiento de los fumadores}

Son varios los problemas que se indican desde los centros en relación al tratamiento de los fumadores, la mayoría de los cuales son los característicos del tratamiento de cualquier adicción. Así, las que incluyen, que presentamos en forma de listado, son:

- Derivación de personas poco motivadas o sin ninguna motivación.

- Percepción por los usuarios de la dificultad que tienen para dejar de fumar.

- Disponer de poco tiempo por sesión (sobre todo para las sesiones individuales) 
- Existencia de psicopatología concomitante.

- Consumo concomitante de otras drogas.

- Problemas a lo largo del tratamiento, como falta de motivación, reticencias a la medicación por el precio, incremento de peso, psicopatología.

- No asistir a los seguimientos.

- Abandonos a lo largo del tratamiento.

- Recaídas.

- Necesidad de mayor formación.

- Falta de medios materiales (ej., cooxímetro), humanos (personal, tiempo) y de fármacos (TSN y bupropión).

\section{Cómo incide dejar de fumar en los pacientes a tra- tamiento por otras drogas}

La mayoría indican que incide de modo positivo. Como ejemplo, indicamos a continuación la opinión de seis de los centros, que son representativos de los que han contestado:

- Centro 1. Positivamente. En relación con el alcohol disminuye el craving mutuo. En pacientes fumadores de hachís, disminuye o elimina el consumo. Mejora los parámetros respiratorios en todos los pacientes.

- Centro 7. De manera muy positiva, aunque existe gran dificultad de abordaje simultáneo de dos drogodependencias a la vez en el caso de tabaco y alcohol, o tabaco y opiáceos porque fundamentalmente el estadio de cambio es distinto.

- Centro 12. Suelen manifestar que las habilidades y estrategias adquiridas, también les son útiles para aplicarlas en el control del consumo de otros tóxicos

- Centro 25. Para muchos adictos a otras drogas dejar de fumar supone un reto difícil de superar. En muchos casos lo consideran un objetivo secundario y no se implican lo suficiente, especialmente cuando advierten lo costoso que les resulta. Por el contrario, el lograrlo, aumenta su autoconfianza y autoestima de forma importante, e incluso adquieren estrategias que les resultan útiles par actuar con otras adicciones. Los familiares de adictos a otras drogas, al dejar de fumar, entienden mejor en que consiste una adicción y esto es favorable para comprender al familiar adicto.

- Centro 29. Es un mayor aumento de la autoestima, la posibilidad de plantearse nuevos retos personales, y se autoafirman en mantener la abstinencia respecto a otras drogas-problema.

- Centro 79. Desde nuestro punto de vista, el dejar de fumar tabaco incide de forma muy positiva, en los tratamientos para la deshabituación de otras drogas por las que esté a tratamiento. La motivación del logro aumenta, y la capacidad de enfrentamiento para enfrentarse a la abstinencia de las otras drogas que consume, la satisfacción personal también es evidente, por lo que también le ayuda para seguir tratando otras adicciones (si he dejado de fumar, que dicen que es lo más duro, también puedo dejar de consumir ...), se refuerza la capacidad de tolerancia y respeto a los demás, ya que pueden ponerse en el lugar del fumador pasivo, lo que también ayuda a su capacidad de convivencia. El control sobre esa conducta, se percibe por parte del paciente, como indicador de posibles controles sobre las demás sustancias, etc.

Con respecto a la familia, también ayuda a ver al paciente como persona más capaz, con lo que el clima familiar es más positivo, iniciándose un cambio relacional y comunicativo que les ayuda a todos.

\section{Características más relevantes de los fumadores que tratan}

Es difícil clasificar globalmente las características de los fumadores que tratan en los distintos centros de drogodependencias y alcoholismo, por la gran variabilidad de los centros, demandas que cubren, área geográfica, etc. Para darnos una idea de esta rica variabilidad, a continuación indicamos la contestación de cinco de estos centros.

- Centro 7. Población rural. Edad media aproximada 40 años. La mayoría tienen estudios primarios, le siguen otros estudios, títulos medios y superiores (un $6 \%$ ). Un $27 \%$ tiene patología dual (depresión, otras drogodependencias,....). Un $27 \%$ tiene problemas respiratorios. Un $20 \%$ problemas cardiacos. Un $20 \%$ patología cancerígena

- Centro 9. Tenemos dos tipos de paciente: 1) alcohólicos en tratamiento: edad media con hepatopatología moderada, y manteniendo actividad laboral y familiar normalizada; 2) paciente para dejar de fumar, sin consumo de otras sustancias, de ambos sexos y derivado desde atención primaria.

- Centro 58. Son pacientes en tratamiento de sustitución con metadona por su dependencia a opiáceos. La/s comorbilidades psiquiátricas más prevalentes se encuentran dentro de los trastornos de la personalidad, si bien los trastornos afectivos, psicóticos y adaptativos están presentes. A nivel físico aproximadamente un $30 \%$ son $\mathrm{VIH}+$ y un $60 \%$ tienen $\mathrm{HVC}+$.

- Centro 79. Normalmente son pacientes adultos, con estudios medios, la mayoría son varones, psicopatología (trastorno límite de personalidad, trastorno 
depresivo recurrente,...), trastornos físicos: hepatitis C, trastorno respiratorios varios.

- Centro 109. En la muestra global desde el año 1997 hasta la actualidad tenemos: el 52,2\% de mujeres, la edad media es de 43,71 años, 86\% con estudios medios o superiores. Variables relativas al tabaquismo: media de cigarrilos/día: 29,52. Media del Test de Fagerström: 6,29. Han realizado intentos previos un 66,2\%, con una media de intentos de 1,20. Antecedentes médicos y psiquiátricos: respiratorios: $34,5 \%$, cardíacos: $24,7 \%$ y psiquiátricos: $33,7 \%$.

\section{Valoración de la inclusión del tratamiento de fu- madores como un servicio más de las Unidades o Centros de Drogodependencias y Alcoholismo para los próximos años}

A la pregunta sobre la valoración que ellos tienen sobre la inclusión del tratamiento de fumadores como un servicio más tenían varias alternativas de respuesta:

a) tratar a las personas que acuden al Centro para el tratamiento de otras drogas,

b) tratar a los fumadores de la población general que quieren dejar de fumar,

c) tratar a otros colectivos (ej., enfermos de atención primaria, personas con enfermedades físicas crónicas y alta dependencia de la nicotina, etc.).

La mayoría de las respuestas son semejantes para cada una de las anteriores alternativas. Como un ejemplo, para la de "tratar a las personas que acuden al Centro para el tratamiento de otras drogas", la mayoria están de acuerdo con ello (85.4\%), otro $8.5 \%$ dice que es necesario pero no lo hacen, un 3.8\% que no lo demanda, y solo un $1.9 \%$ dice que no.

\section{Valoración global del tratamiento de fumadores en tu Centro}

Las valoraciones globales que han hecho en cada centro describen la mayoría de los datos anteriores, desde la satisfacción con el tratamiento que aplican, en unos casos, a la inexistencia de tratamientos en otros, y comentarios diversos. Para darnos una idea de sus respuestas, una muestra aleatoria de los mismos aparece a continuación, de entre los que han contestado al cuestionario.

- Centro 1. En estos momentos estamos reelaborado el programa de asistencia incluyendo la evaluación sistemática del mismo.

- Centro 10. Mi valoración global es positiva. Si lo comparamos con el tratamiento para otras drogas nuestra experiencia no es amplia. Si pudiéramos disponer de material (información, carteles, cooxímetros...) se podría mejorar.

- Centro 30. Prácticamente no se realizan tratamientos. Creo que lo más eficaz y eficiente en tabaquismo es el tratamiento grupal, que yo no puedo ofertar sino cuento con más recursos humanos o colaboración externa.

- Centro 70. El tratamiento es efectivo pero tenemos pendiente, por cuestiones de tiempo, la protocolización por escrito del mismo.

- Centro 80. Creo que la demanda es muy baja, porque se siguen viendo a las UADs como referencia en el tratamiento de sustancias toxicas, heroína; empezamos a recibir consumos de psicoestimulantes como única droga, cannabis y alcohol. Todavía no se acepta el tabaco como similar a las anteriores sustancias.

- Centro 100. No se realiza. Tendríamos que estar más sensibilizados en este abordaje.

- Centro 110. Creo que podemos estar contentos de nuestro trabajo, que como unidad especializada dentro de la atención primaria también tiene su ubicación como centro de drogodependencias. Quizás la falta mas importante que tenemos es la infraestructura informática que es muy deficitaria y nos impide realizar todas les actividades de la manera que desearíamos.

\section{Perspectivas de futuro que ven para el tratamiento de fumadores en los Centros y Unidades de Drogo- dependencias y Alcoholismo}

Una cuestión de gran relevancia, junto al conocimiento de la situación actual, es saber cuál es la perspectiva de futuro que ven los profesionales de los centros de drogodependencias y alcoholismo para el tratamiento de los fumadores. En este estudio se les preguntó esta cuestión referida a cómo lo ven para los próximos cinco años.

Los resultados indican que mayoritariamente los centros de drogodependencias ven que el tratamiento de fumadores tiene buenas o muy buenas perspectivas de futuro $(29,1 \%)$ y que va a haber un aumento de la demanda (24,3\%) (ver tabla 16). Pero también hay otras respuestas que van en la línea de que precisan más medios para llevar a cabo la asistencia que produce la demanda, que el tratamiento en los centros depende de las directrices de cada comunidad autónoma, etc. También destaca, aunque con porcentaje menores, aquellos que no lo ven viable $(6.8 \%)$ o que se hará en otros dispositivos. 
Tabla 16. Perspectivas de futuro que indican los centros sobre el tratamiento de fumadores para los próximos años

$\begin{array}{lrr}\text { Respuesta } & \mathbf{n} & \text { \% } \\ \text { Buenas, muy buenas } & 30 & 29,1 \\ \text { Aumento de la demanda } & 25 & 24,3 \\ \text { No, no lo veo } & 7,8 \\ \text { Viable, siempre que no se dispare la demanda o se aumente el personal } & 6 & 6,8 \\ \text { Dependerá de las directrices de los Planes Autonómicos de Drogas } & 4,8 \\ \text { Hay que integrar el tratamiento de los fumadores en los centros de drogodependencias } & 3,9 \\ \text { Escasa demanda, disminuye la demanda en los centros } & 3,9 \\ \text { Depende de varios factores (ej., integración) } & 2 \\ \text { Intervención tras fracasos previos (ej., primaria, otros) } & 2 \\ \text { Incierto } & 2 \\ \text { Ya está integrado en los centros } & 1,9 \\ \text { Ninguna si no se aumenta el personal } & 1,9 \\ \text { Se hará en otros dispositivos distintos a nuestros centros } & 1,9 \\ \text { Otros } & 1 \\ \text { Total } & 1,0 \\ \end{array}$

\section{DISCUSIÓN}

El tratamiento del tabaquismo es un servicio más que prestan muchos de los centros de drogodependencias y unidades de alcoholismo en España, según los datos de la muestra que hemos obtenido en este estudio de 142 centros de 11 comunidades autónomas. Los datos indican que a lo largo del año 2004, más de la mitad de los centros (un 55.6\%) llevan a cabo sistemáticamente tratamientos para los fumadores que acuden a ellos, bien sean sus propios usuarios (dependientes de la heroína, cocaína, etc.) o bien personas que les demandan directamente tratamiento para dejar de fumar en sus centros. De estos que han contestado (70 centros) han tenido demanda de tratamiento de 3.771 personas fumadoras, de éstas evaluaron a 3.109 y trataron en torno a 3.000. De éstas finalizaron el tratamiento en torno a la mitad (de los 55 centros que contestaron a esta pregunta finalizaron el tratamiento 1.355). La mayoría de los centros están tratando pocos fumadores, con excepciones de tratamientos ya amplios de más de 100 fumadores por centro al año, y el centro con más fumadores tratados fue de 515 fumadores a lo largo del año

El porcentaje de abstinencia que obtienen los distintos centros es difícil de evaluar por la falta de datos en muchos de los centros. El peso asistencial impide a muchos centros disponer de adecuados registros para saber en poco tiempo la eficacia de sus intervenciones. Por otra parte, al no estar el tratamiento de fumadores siempre disponible como servicio normalizado del centro, o no estar protocolizada la recogida de datos, o por las dificultades y criterios a utilizar para decir donde hay y no hay un criterio de abstinencia o éxito, hace difícil tener datos en la línea de una investigación científica. Esta no es la función específica de los centros, de ahí que no siempre tengan este tipo de datos. Pero, cuando hay datos, los resultados son buenos, en comparación con los estudios clínicos publicados del tratamiento del tabaquismo, ya que en el $38 \%$ de los centros el nivel de abstinencia al final está entre el 41 y el $50 \%$ de éxito, y otro adicional $29 \%$ de los centros indican que tienen niveles de eficacia entre el 51 y el $100 \%$. Como es bien sabido en cualquier adicción, este nivel desciende en los seguimientos. A los seis meses la mayoría de los centros que contestan indican que el nivel de abstinencia de sus fumadores está por debajo del 50\%. También a los 12 meses la mayoría de los porcentajes de abstinencia están por debajo del $50 \%$ aunque han contestado muy pocos centros para los 12 meses.

La limitación de los datos del presente estudio para los seguimientos de 6 y 12 meses está en que pocos centros realizan seguimientos y pocos han contestado. Una sugerencia para el futuro es que aquellos centros que traten 100 o más fumadores al año, o cuando varios centros puedan tener entre ellos 100 o más fumadores al año tratados, hagan seguimientos para comprobar el mantenimiento de los buenos resultados que obtienen al final del tratamiento la mayoría de ellos. Lo cierto es que los niveles de abstinencia indicados en este estudio son muy buenos, para lo que es habitual en adicciones y en el tratamiento del tabaquismo (Sancho et al., 2003), aunque por otro lado son lógicos ya que en estos centros están los profesionales mejor capacitados para tratar una adicción, sea la 
dependencia de la heroína, cocaína, cannabis, alcohol, nicotina u otras.

También hay que indicar, sobre los datos anteriores, que la abstinencia va a depender del tipo de paciente tratado (si con dependencia de heroína, cocaína, cannabis, alcoholismo, población general, remitido por atención primaria, etc.). En este sentido el perfil del usuario de estos centros no es posible establecerlo por su variabilidad, características de cada centro, personas que atienden, tipo de usuarios, lugar geográfico, tipo de profesionales, motivación para tratar fumadores, etc.

Los datos indican que el tratamiento que más se utiliza es el combinado de tratamiento psicológico más farmacológico, seguido del tratamiento psicológico y en algunos casos, menos, solo farmacológico. Esto va en la línea de utilizar tratamientos eficaces y validados empíricamente (Becoña, 2004b). En la mayoría de los centros son los distintos profesionales del centro los que participan en el tratamiento o en las distintas fases del mismo, aunque hay un peso relativo mayor del psicólogo en llevar a cabo el tratamiento, sea individualmente o en grupo, o de aplicarlo él solo en algunos centros.

Los profesionales encuentran toda una serie de limitaciones para la aplicación de los tratamientos a los fumadores, típicas de las adicciones (ej., falta de motivación, abandonos del tratamiento, recaídas), aunque las mayores están en la falta de suficiente tiempo, carencia de personal, incremento de la demanda, etc. Mayoritariamente, el $94 \%$ de los que han contestado, indican que el tratamiento de fumadores debe hacerse en los centros de drogodependencias, tanto para los usuarios habituales de estos centros, como para fumadores de la población general que lo demanden, como de otros fumadores que les deriven de primaria, hospitales, etc. Pero al mismo tiempo se aprecia en un porcentaje importante de centros las limitaciones y dificultades que esto está teniendo y seguirá teniendo en el día a día asistencial si la Administración no asume claramente el tratamiento de la dependencia de la nicotina en estos centros, si no le aporta suficientes medios (de personal fundamentalmente) y si no clarifican las derivaciones y papel de los distintos recursos que existen para tratar a los fumadores (atención primaria, hospitales, unidades especializadas, etc.).

La conclusión final de este estudio es que los centros de drogodependencias y alcoholismo son un punto de referencia en España para el tratamiento de fumadores, como lo muestra el que no menos de 3.000 personas fueron tratadas en el año 2.004 en los centros que han contestado al cuestionario remitido, lo que sugiere que la cifra real de fumadores tratados en los centros de drogodependencias y alcoholismo es mucho mayor. De hacer el estudio en todos este número se incrementaría considerablemente. De todos modos, con el incremento de la demanda que se viene produciendo, esta cifra seguirá creciendo año a año. La eficacia de las intervenciones, la profesionalidad de los clínicos de estos centros, y su extensa distribución en todas las comunidades autónomas, indica que los centros de drogodependencias y alcoholismo vienen cumpliendo y seguirán cumpliendo un importante papel en el tratamiento de las personas con dependencia de la nicotina, como lo vienen haciendo desde hace ya varias décadas en las drogas ilegales y alcoholismo.

\section{AGRADECIMIENTOS}

Este estudio ha sido financiado a Socidrogalcohol por la Delegación del Gobierno para el Plan Nacional sobre Drogas.

\section{REFERENCIAS}

American Psychiatric Association (2000). Diagnostic and statistical manual for mental disorders, 4th edition, revised text. Washington, DC: American Psychiatric Association (traducción castellana en Barcelona, Massón, 2002).

Banegas, J.R., Díez, L., González, J., Villar, F. y RodríguezArtalejo (2005). La mortalidad atribuible al tabaquismo comienza a descender en España. Medicina Clínica, 124, 769-771.

Becoña, E. (2004a). Guía clínica para ayudar a los fumadores a dejar de fumar. Palma de Mallorca: Socidrogalcohol.

Becoña, E. (2004b). Tratamiento psicológico del tabaquismo. Adicciones, 16 (Supl. 2), 237-263.

Echave, J. de, Flordelís, C., García, V., Riba, M.V. de la, Llopis, J.J. y Martín, E. (2004). El tratamiento del tabaquismo en los centros de drogodependencias y unidades de conductas adictivas. Adicciones, 16 (Supl. 2), 321-337.

Gurrea, A. y Pinet, M.C. (2004). Tabaco y patología afectiva. Adicciones, 16 (Supl. 2), 155-176.

Herrero, F.J. y Moreno, J.J. (2004). Tratamientos combinados del tabaquismo: psicológicos y farmacológicos. Adicciones, 16 (Supl. 2), 287-320.

Jiménez, L., Bascarán, M.T., García-Portilla, M.P., Sáiz, P.A., Bousoño, M. y Bobes, J. (2004). La nicotina como droga. Adicciones, 16 (Supl. 2), 143-153.

Martínez, J.M., Gurpegui, M., Díaz, F.J. y León, J. de (2004). Tabaco y esquizofrenia. Adicciones, 16 (Supl. 2), 177190.

Mateu, G., Astals, M. y Torréns, M. (2005). Comorbilidad psiquiátrica y trastorno por dependencia de opiáceos: del diagnóstico al tratamiento. Adicciones, 17 (Supl. 2), $111-121$. 
Ministerio de Sanidad y Consumo (2005). Encuesta nacional de salud 2003. Madrid: Ministerio de Sanidad y Consumo.

Montes, A., Pérez, M. y Gestal, J.J. (2004). Impacto del tabaquismo sobre la mortalidad en España. Adicciones, 16 (Supl. 2), 75-82).

Nerín, I. y Córdoba, R. (2004). Tratamiento farmacológico. Terapia sustitutiva con nicotina. Adicciones, 16 (Supl. 2), $265-274$.

Nieva, G., Gual, A., Ortega, L. y Mondón, S. (2004). Alcohol y tabaco. Adicciones, 16 (Supl. 2), 191-199.

Plan Nacional sobre Drogas (2003). Observatorio español sobre drogas. Informe 6. Madrid: Delegación del Gobierno para el Plan Nacional sobre Drogas.

Plan Nacional sobre Drogas (2004). Datos de la encuesta escolar y la de población general. Madrid: Delegación del Gobierno para el Plan Nacional sobre Drogas.
Sáiz, P.A., García-Portilla, M.P., Martínez, S., Bascarán, M.T., Paredes, B. y Bobes, J. (2004). Tratamiento farmacológico. Psicofármacos. Adicciones, 16 (Supl. 2), 275-285.

Salvador, T. (1996). Tabaquismo. Madrid: Aguilar.

Sancho, J.L. de, Gorgojo, L., González, J. y Salvador, T. (2003). Evaluación de la eficacia, efectividad y costeefectividad de los distintos abordajes terapéuticos para dejar de fumar. Informe de Evaluación de Tecnologías Sanitarias $n^{\circ}$ 40. Madrid: Agencia de Tecnologías Sanitarias. Instituto de Salud Carlos III. Ministerio de Sanidad y Consumo.

Santo-Domingo, J. (2002). Introducción. Evolución del alcoholismo y su asistencia en España. Adicciones, 14 (Supl. 1), 7-21. 
